Journal of Mathematics and Statistics 2 (4): 453-456, 2006

ISSN 1549-3644

(C) 2006 Science Publications

\title{
On Self-similar Solution of Non-linear Diffusion Equation with Convection Term
}

${ }^{1}$ B. I .Olajuwon and ${ }^{2}$ A.O. Popoola

${ }^{1}$ Department of Mathematics, University of Agriculture, Abeokuta Ogun State, Nigeria

${ }^{2}$ Department of Pure and Applied Mathematics, Ladoke Akintola University of Technology

Ogbomoso, Oyo State, Nigeria

\begin{abstract}
We consider the self-similar solution of non-linear diffusion equation with convection term. We examine the existence and uniqueness of solution of the problem. The numerical result is presented as concentration profile for various values of $\mathrm{m}$, the diffusion index.
\end{abstract}

Key words: Diffusion equation, convection term

\section{INTRODUCTION}

The study of solution of mathematical equation describing diffusion problems has over the years attracted the interest of many researchers. Cherniha and Serov ${ }^{[1]}$ considered the Lie and Non-lie symmetries of non-linear diffusion equation with convection term. They presented new ansätze and exact solution for the non-linear diffusion equations.

Kuske and Mileniski ${ }^{[2]}$ derived new modulation equations for hexagonal patterns in reaction-diffusion systems. These systems include additional nonlinearities, which are not present in Rayleigh-Bernard convection or Smith-Hohenberg type models. The investigation demonstrates instabilities and a new phenomenon not found in other systems, and is applied to patterns of flame fronts in certain models of burnerstabilized flames.

Nakamura et $a l^{[3]}$ studied reaction diffusion equations with a spatially inhomogeneous reaction term. If the coefficient of this reaction term is much larger than the diffusion coefficient, a sharp interface appears between two different phases. They showed that the equation of motion of such interface involves a drift term despite the absence of drift in the original diffusion equations. In particular, they showed that the same rich spatial patterns observed for Chemotaxisgrowth model can be realized by a system without a drift term.

Ayeni and Agusto ${ }^{[4]}$ investigated the existence and uniqueness of solution of self-similar diffusion equation. The paper examined the models of fast flow of gas in porous medium and microwave heating of various materials.

Popoola et al. ${ }^{[5]}$ investigated a system of reaction diffusion problem with a characteristic diffusion term. They presented the similarity solution of the problem and stated the condition for the existence of similarity solution. They discussed the effect of the diffusion parameter on the reaction.

In this paper, we consider the self-similar solution of non-linear diffusion equation with convection term.
Of particular interest is the question of existence and uniqueness of the solution. We also present the numerical result as a concentration profile for various values of $\mathrm{m}$, the diffusion index.

Mathematical problem: We consider the non-linear diffusion equation of the form

$$
\frac{\partial u}{\partial t}-A(u) \frac{\partial^{2} u}{\partial x^{2}}=\left[B(u)+\frac{\partial A(u)}{\partial x}\right] \frac{\partial u}{\partial x}+C(u)
$$

With the boundary conditions

$u(0, t)=U, u(\infty, t)=0$

Where

$$
\begin{aligned}
& A(u)=\lambda_{0} e^{m u} \\
& B(u)=-\frac{\lambda_{0}{ }^{2} u^{m} e^{m u}}{t^{1 / 2}} \\
& C(u)=\frac{\lambda_{0} u^{2 m+1}}{t} \quad, m>0
\end{aligned}
$$

$u(x, t)$ is the concentration, $\mathrm{m}$ is the diffusion index and $\lambda_{0}$ is an arbitrary constant.

\section{Remark 1:}

Equation (1) reduces to classical heat equation, if $\mathrm{A}=1$, $\mathrm{B}=\mathrm{C}=0$.

Equation (1) reduces to non-linear heat equation, if $\mathrm{B}=\mathrm{C}=0$.

Equation (1) reduces to non-linear equation with a source term, if $\mathrm{B}=0$.

Similarity transformation: Define a similarity variable $\eta$, as

$\eta=\frac{x}{t^{\alpha}}$

Such that

$u(x, t)=U f(\eta)$

Using equations (3), (4), (5), (6) and (7) in equation (1) then equation (1) together with the boundary condition (2) becomes

Corresponding Author: B. I. Olajuwon, Department of Mathematics, University of Agriculture, Abeokuta Ogun State, Nigeria 
$f^{\prime \prime}+\left(\frac{\eta e^{-m f}}{2 \lambda_{0}}-\lambda_{0} f^{m}\right) f^{\prime}+m f^{\prime 2}+e^{-m f} f^{2 m+1}=0$

$f(0)=1, \quad f(\infty)=0$

Remark 2: Self-similar solution exists for $\alpha=\frac{1}{2}$

Existence and uniqueness

Theorem 1: For each $m>0$, equation (1) with the boundary condition (2) has a unique solution.

Remark 3: The existence and uniqueness of solution of problem (8) satisfying

Boundary condition (9) implies the existence and uniqueness of solution of problem (1) satisfying boundary condition (2). Resolve problem (8) into a system of equations

Let

$$
\left(\begin{array}{l}
y_{1} \\
y_{2} \\
y_{3}
\end{array}\right)=\left(\begin{array}{l}
\eta \\
f \\
f^{\prime}
\end{array}\right)
$$

So we consider

$$
\left(\begin{array}{l}
y_{1}^{\prime} \\
y_{2}^{\prime} \\
y_{3}^{\prime}
\end{array}\right)=\left(\begin{array}{l}
1 \\
y_{3} \\
\left(\lambda_{0} y_{2}^{m}-\frac{y_{1} e^{-m y_{2}}}{2 \lambda_{0}}\right) y_{3}-m y_{3}^{2}-e^{-m y_{2}} y_{2}^{2 m+1}
\end{array}\right)
$$

Together with the initial conditions,

$$
\begin{aligned}
& \left(\begin{array}{l}
y_{1}(0) \\
y_{2}(0) \\
y_{3}(0)
\end{array}\right)=\left(\begin{array}{l}
0 \\
1 \\
-\beta
\end{array}\right) \\
& 0 \leq y_{1} \leq \infty, 1 \leq y_{2} \leq L,-\beta \leq y_{3} \leq 0
\end{aligned}
$$

Where $\beta$ is guessed such that the boundary condition (9) is satisfied. $L, \beta$ are positive constants.

Remark 4: Problem (1) can be re-written as

$$
\left(\begin{array}{l}
y_{1} \\
y_{2} \\
y_{3}
\end{array}\right)=\left(\begin{array}{l}
f_{1}\left(y_{1}, y_{2}, y_{3}\right) \\
f_{2}\left(y_{1}, y_{2}, y_{3}\right) \\
f_{3}\left(y_{1}, y_{2}, y_{3}\right)
\end{array}\right)
$$

Theorem 2: For $\mathrm{m}>0, \lambda_{0}>0$, problem (11) satisfying the initial conditions (12) is Lipschitz continuous.

Proof: By remark (4), equation (11) can be written as (14).

Clearly, $\frac{\partial f_{i}}{\partial y_{j}}, \quad i, j=1,2,3$ are bounded and so, there exists a constant $\mathrm{k}>0$

Such that

$k=\max \left|\frac{\partial f_{i}}{\partial y_{j}}\right|, \quad i, j=1,2,3$ Where $\mathrm{k}$ is the Lipschitz constant. Hence $f_{i}\left(y_{1}, y_{2}, y_{3}\right), i, j=1,2,3$ is Lipschitz continuous and so, problem (11) satisfying the initial condition (12) is Lipschitz continuous.

Remark 5: The system of equations (14) can also be re-written as

$Y^{\prime}=F(\eta, Y)$

and the initial condition (12) is written as

$Y(0)=Y_{0}$

Theorem 3: Let $\mathrm{m}>0, \lambda_{0}>0$, suppose $F(\eta, Y)$ is continuous and satisfy the Lipschitz condition $\|F(\eta, Y)-F(\eta, X)\| \leq L\|Y-X\|$

For all $X, Y \in B=\left\{Y \in R^{n} /\left\|Y-Y_{0}\right\| \leq r\right\}$

Then, there exist $\delta>0$ such that problem (15) satisfying initial condition (16) has a unique solution $\operatorname{over}\left[\eta_{0}, \eta_{0}+\delta\right]$ where $\left[\eta_{0}, \eta_{0}+\delta\right] \subset[0, \infty]$.

Proof: By (15)

$Y(\eta)=Y_{o}+\int_{\eta_{o}}^{\eta} F(s, Y(s)) d s$

Define a mapping $Y:\left[\eta_{0}, \eta_{1}\right] \rightarrow R^{n}$ and denote it by $(U Y)(\eta)$, we write $(17)$ as

$Y(\eta)=(U Y)(\eta)$

where $(U Y)(\eta)$ is continuous in $\eta$.

Define a Banach space $M$ and a closed set $S \subset M$ such that $\mathrm{U}$ maps $\mathrm{S}$ into $\mathrm{S}$ and is contraction over $\mathrm{S}$.

Let,

$M=C\left[\eta_{0}, \eta_{0}+\delta\right]$

with norm

$\|Y\|_{C}=\max _{\eta \in\left[\eta_{0}, \eta_{0}+\delta\right]}\|Y(\eta)\|$

and

$S=\left\{Y \in M /\left\|Y-Y_{0}\right\|_{C} \leq r\right\}$

Where $\mathrm{r}$ is the radius of the ball B and $\delta>0$.

Clearly, $\|Y(\eta)\|$ denotes a norm on $R^{n}$ and $\|Y\|_{C}$ denotes a norm on $\mathrm{M}$.

Also, $\mathrm{B}$ is a ball in $\mathrm{R}^{\mathrm{n}}$ and $\mathrm{S}$ is a ball in $\mathrm{M}$. By definition, $U$ maps $M$ into $M$. We shall show that $U$ maps $\mathrm{S}$ into $\mathrm{S}$.

Equation (17) and (18) imply that,

$(U Y)(\eta)-Y_{0}=\int_{\eta_{0}}^{\eta} F(s, Y(s)) d s$

$=\int_{\eta_{0}}^{\eta}\left[F(s, Y(s))-F\left(s, Y_{0}\right)+F(s, Y(s))\right] d s$

By the virtue of theorem 2, $F(\eta, Y)$ is bounded on $\left[\eta_{0}, \eta_{1}\right]$. Let,

$$
k=\max _{\left.\eta \in\left[\eta_{0}, \eta_{1}\right]\right]}\left\|F\left(\eta, Y_{0}\right)\right\|
$$


Using the Lipschitz condition and the fact that for each $Y \in S$,

$$
\left\|Y(\eta)-Y_{0}\right\| \leq r, \forall \eta \in\left[\eta_{0}, \eta_{0}+\delta\right]
$$

We obtain,

$$
\begin{aligned}
& \left\|(U Y)(\eta)-Y_{0}\right\| \leq \int_{\eta_{0}}^{n}[\|F(s, Y(s))-F(s, Y(s))\|+\| F(s, Y(s) \|] d s \\
& \leq \int_{\eta_{0}}^{n}\left[L\left\|Y(s)-Y_{0}\right\|+k\right] d s \\
& \leq \int_{\eta_{0}}^{\eta}[L r+k] d s \\
& \leq\left(\eta-\eta_{0}\right)(L r+k)
\end{aligned}
$$

And$$
\left\|U Y-Y_{0}\right\|_{C}=\max _{\eta \in\left[\eta_{0}, \eta_{0}+\delta\right]}\left\|(U Y)(\eta)-Y_{0}\right\|
$$$$
\leq \delta(L r+k)
$$

Therefore choosing

$$
\delta \leq \frac{r}{(L r+k)}
$$

ensures $\mathrm{U}$ maps $\mathrm{S}$ into $\mathrm{S}$.

Next, we show that $\mathrm{U}$ is a contraction mapping over $\mathrm{S}$. Let $X$ and $Y \in S$ and consider,

$$
\begin{aligned}
& \|(U Y)(\eta)-(U X)(\eta)\|=\left\|\int_{\eta_{0}}^{\eta}[F(s, Y(s))-F(s, X(s))] d s\right\| \\
& \leq\left\|\int_{\eta_{0}}^{\eta} F(s, Y(s))-F(s, X(s)) d s\right\| \\
& \leq\left\|\int_{\eta_{0}}^{\eta} L\right\| Y(s)-X(s)\|d s\| \\
& \leq\left\|\int_{\eta_{0}}^{\eta} L\right\| Y-X\|d s\|_{C}
\end{aligned}
$$

Therefore,

$\|U X-U Y\|_{C} \leq L \delta\|Y-X\|_{C} \leq \rho\|Y-X\|_{C}$,

for $\delta \leq \frac{\rho}{L}$

Choosing $\rho \leq 1$, and $\delta \leq \frac{\rho}{L}$. then, clearly $\mathrm{U}$ is a contraction mapping over $\mathrm{S}$

Choosing,

$$
\delta \leq \min \left\{\eta_{1}-\eta_{0}, \frac{r}{L r+k}, \frac{\rho}{L}\right\}, \text { for } \rho<1
$$

Then, (17) has a unique solution in $\mathrm{S}$.

Thus since any solution of (17) in M will lie in S. So, the uniqueness of the solution in $\mathrm{S}$ implies uniqueness in M. Hence, problem (15) has a unique solution.

Proof of theorem 1: The uniqueness of solution of problem (15) implies the uniqueness of solution of problem (11). The uniqueness of solution of problem
(11) implies the uniqueness of solution of problem (8). Also the uniqueness of solution of problem (8) implies the uniqueness of solution of problem (1) satisfying condition (2). This completes the proof.

Numerical solution: Resolving problem (8) into system of equations we have,

$$
\left(\begin{array}{l}
y_{1}^{\prime} \\
y_{2}^{\prime} \\
y_{3}^{\prime}
\end{array}\right)=\left(\begin{array}{l}
1 \\
y_{3} \\
\left(\lambda_{0} y_{2}^{m}-\frac{y_{1} e^{-m y_{2}}}{2 \lambda_{0}}\right) y_{3}-m y_{3}{ }^{2}-e^{-m y_{2}} y_{2}{ }^{2 m+1}
\end{array}\right)
$$

Together with the initial conditions,

$$
\left(\begin{array}{l}
y_{1}(0) \\
y_{2}(0) \\
y_{3}(0)
\end{array}\right)=\left(\begin{array}{l}
0 \\
1 \\
-\beta
\end{array}\right)
$$

Where $\beta$ is guessed such that the boundary condition (9) is satisfied. The numerical result is presented in the figure below as concentration profile.

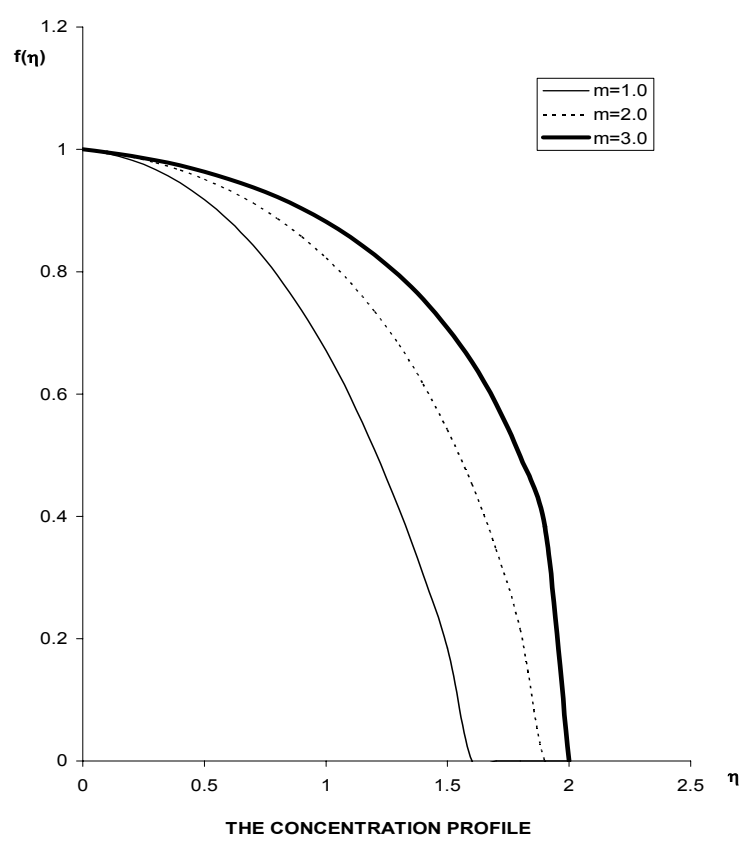

\section{RESULTS AND DISCUSSION}

The existence and uniqueness of solution of the non-linear diffusion problem has been established in theorems (1), (2) and (3). The fact that the problem has a unique solution showed that the mathematical model (1) represents a physical situation. The concentration profile is as shown in the figure. It is clearly shown from the figure that the system observed a high rate of diffusion with low diffusion index $\mathrm{m}$. This study has greater application in many engineering and scientific processes. 


\section{ACKNOWLEDGEMENT}

The authors appreciate the useful suggestions, contributions and encouragements provided by Professor R.O.Ayeni.

\section{REFERENCES}

1. Cherniha, R and M. Serov, 1997. Lie and non-lie symmetries of non-linear diffusion equations with convection term. Symmetry in Non-linear Mathematical Physics, 2: 444-449.

2. Kuske, R. and P. Milemski, 1999. Modulated twodimensional patterns in reaction- diffusion systems. European J. Appl. Math., 10: 157-184.

3. Nakamura, K.-I, H. Matano, D. Hilhorst and R. Schaätzle, 1999. Singular limit of a reaction diffusion equation with a spatially inhomogeneous reaction term. J. Statist. Phys., 95: 1165-1185.
4. Ayeni R.O. and F.B. Agusto, 2000. On the existence and uniqueness of self-similar diffusion equation. J. Nig. Ass. Math. Phys., 4: 183.

5. Popoola, A.O., B.I. Olajuwon and P.O. Olanrewaju, 2003. Effect of a diffusive parameter on a reaction diffusion problem. J. Math. Ass. Nig. (ABACUS), 30: 61-65. 